\title{
Unified approach for the explanation of stochastic and periodic moirés
}

\author{
Isaac Amidror \\ Ecole Polytechnique Fédérale de Lausanne \\ Laboratoire de Systèmes Périphériques \\ 1015 Lausanne \\ Switzerland
}

\begin{abstract}
Moiré phenomena of different types are frequently encountered in electronic imaging. Most common are moiré effects that occur between periodic structures. These effects have been intensively investigated in the past, and their mathematical theory is today fully understood. The same is true for moiré effects between repetitive layers (i.e., between geometric transformations of periodic layers). However, although moiré effects that occur between random layers (Glass patterns) have long been recognized, only little is known today about their mathematical behavior. In this work we study the behavior of such moirés, and compare it with analogous results from the periodic case. We show that all cases, periodic or not, obey the same basic mathematical rules, in spite of their different visual properties. This leads us to a unified approach that explains both the behavior of Glass patterns in the stochastic case, and the well-known behavior of the moiré patterns in periodic or repetitive cases. (C) 2003 SPIE and IS\&T. [DOI: 10.1117/1.1604785]
\end{abstract}

\section{Introduction}

The moire effect is a well-known phenomenon that occurs when two or more structures such as gratings, screens, etc., interfere with each other and generate a new visible pattern that does not exist in any of the original structures (Fig. 1).

Moiré phenomena are frequently encountered in electronic imaging. For example, in the field of color printing, three or four halftone dot screens must be superposed, one for each of the primary color inks being used (usually cyan, magenta, yellow, and black). ${ }^{1}$ When these halftone screens are made of periodic dot screens, strong undesirable periodic moiré effects may occur in some circumstances between the superposed screens (Ref. 2, Sec. 3.3). The use of random dot screens instead of periodic dot screens is known to be an efficient solution to this problem, since random screens do not generate in their superposition such moire effects. This is particularly helpful in nonstandard color printing when the number of color inks being used (and thus, the number of halftone screens) exceeds four, in which case it becomes extremely difficult (if at all possible) to find moiré-free combinations of periodic screens.

On the other hand, it is also known that the superposition of aperiodic layers such as random dot screens may

Paper 02104 received Oct. 24, 2002; revised manuscript received Apr. 13, 2003; accepted for publication May 27, 2003.

$1017-9909 / 2003 / \$ 15.00$ @ 2003 SPIE and IS\&T. give rise to a different type of moiré pattern, which consists of a single structure resembling a top-viewed funnel, or a distant galaxy in the night sky [see Fig. 2(b)]. This phenomenon is known in literature as a Glass pattern, after Leon Glass who described it in the late 1960s. ${ }^{3-5}$ However, Glass patterns are still much less understood today than periodic or repetitive moiré effects, partly because they do not easily lend themselves to the same mathematical tools that so nicely explain the classical moiré effects between periodic or repetitive layers.

In the present work we present a general, unified approach that explains all of these phenomena. We show that in spite of their completely different visual appearance, moirés between periodic or aperiodic layers are in fact particular cases of the same phenomenon, and they all follow the same fundamental rules that explain what happens in the superposition of any layers, periodic or not. In particular, we show the rules that govern the existence or inexistence of moiré phenomena, and their behavior under layer mappings (layer rotations, scalings, shifts, etc.).

We start in Sec. 2 by establishing the terminology and the basic notions that are needed for the rest of the work. In Sec. 3 we discuss the superposition of aperiodic layers and

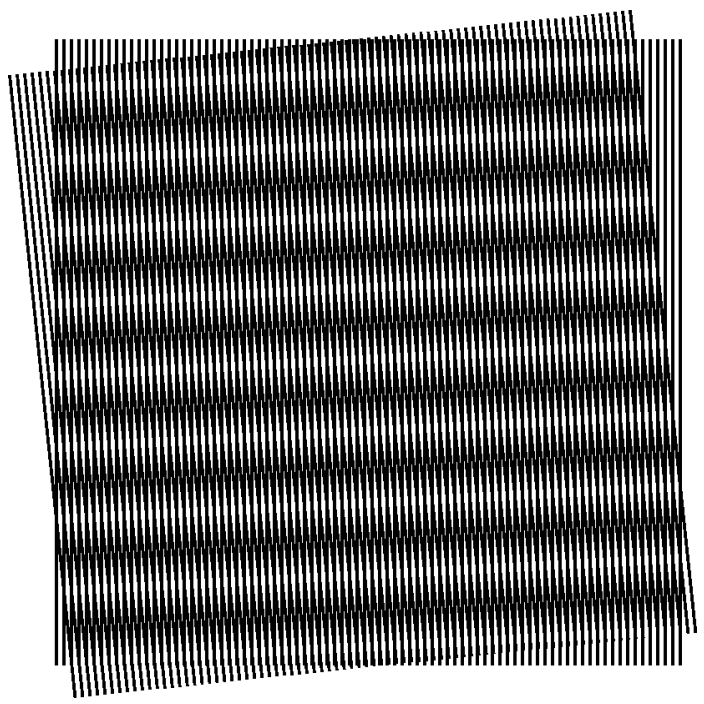

Fig. 1 An example of a moire effect that occurs due to the superposition of two identical periodic layers with a small angle difference. 


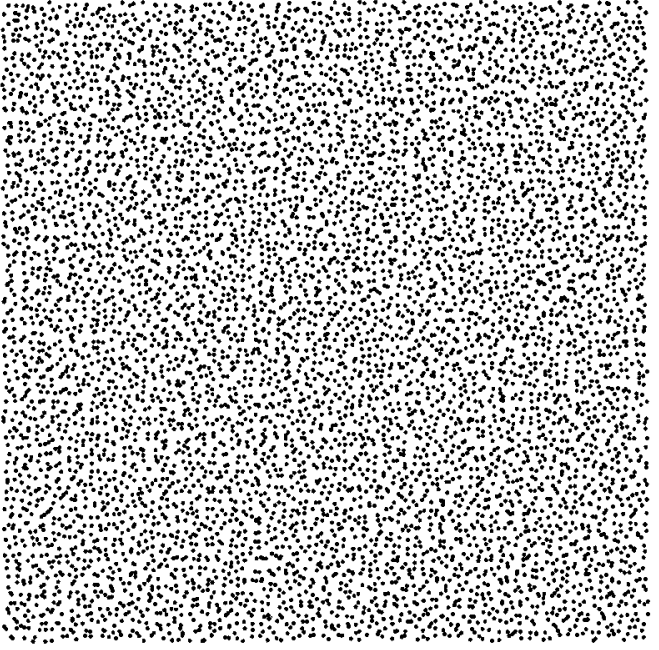

(a)

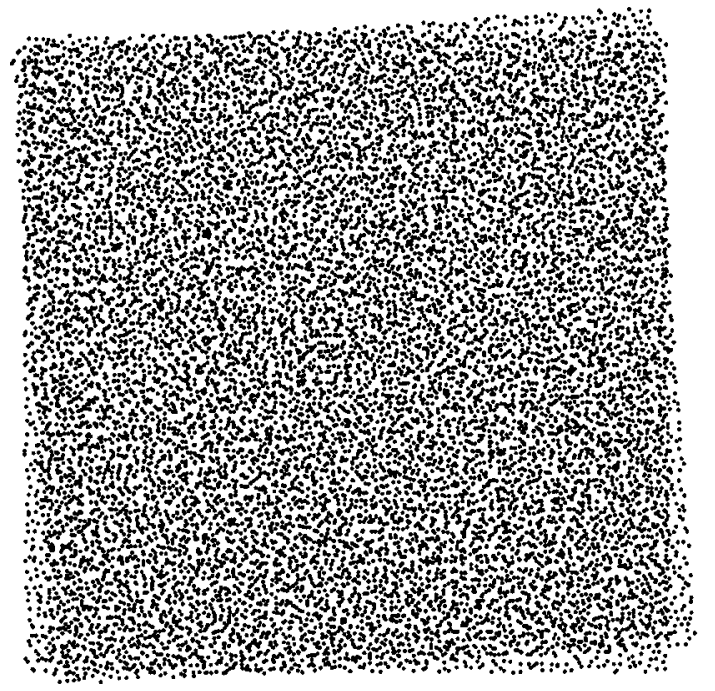

(c)

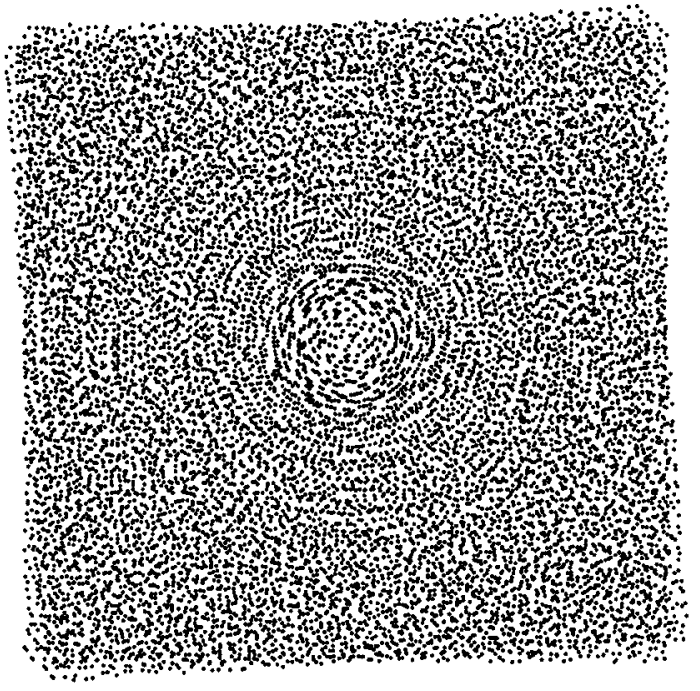

(b)

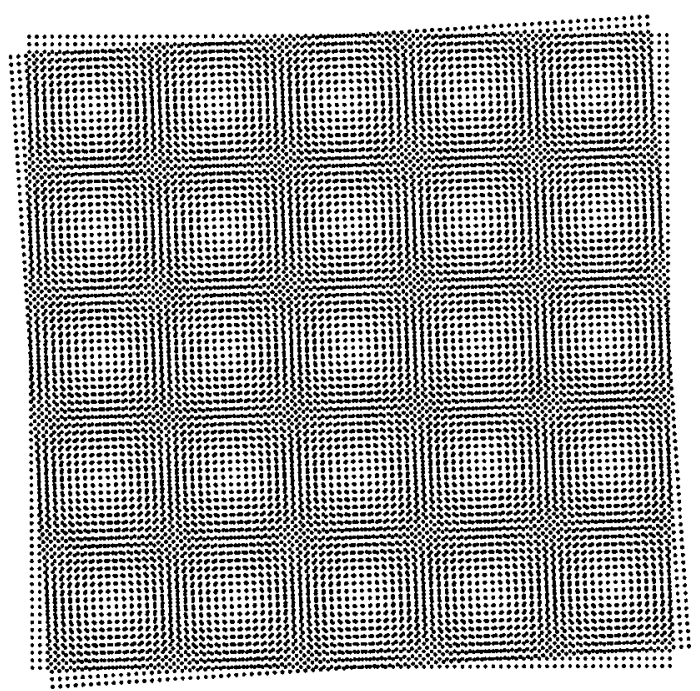

(d)

Fig. 2 (a) An aperiodic dot screen. (b) The superposition of two identical copies of the aperiodic dot screen (a) with a small angle difference gives a moire effect in the form of a Glass pattern around the center of rotation. However, if one of the aperiodic layers is rotated by $180 \mathrm{deg}$, as shown in (c), the Glass pattern disappears. (d) When the superposed layers are periodic, a Glass pattern is still generated around the center of rotation, but due to the periodicity of the layers, this pattern is periodically repeated throughout the superposition, thus generating a periodic moiré pattern.

explain its main properties; this leads us to a general rule that determines the moire existence conditions for all cases, periodic or not. Then, in Sec. 4, we explain the mathematical meaning of Glass patterns, using the fixed point theorem and its particular case for affine transformations. In Sec. 5 we explain the behavior of Glass patterns under layer mappings, and show that the behavior of the corresponding periodic moires is in fact just the same. This leads us to a second general rule, which determines the influence of layer mappings (rotation, scaling, layer shifts, etc.) on the moire in both periodic and aperiodic cases. Finally, in Sec. 6 we present the main conclusions.

\section{Background and Basic Notions}

In this introductory section, we briefly review the basic notions and terminology that are used later. Since we deal with layers and layer superpositions throughout this work, let us start by explaining these notions and their main properties. In fact, a layer (or image) is the most general term 
we use to cover anything in the image domain. It can be periodic or not, continuous or binary, etc. However, we still need to make some basic assumptions on our layers.

First of all, we limit ourselves here to monochrome, black and white images. This means that each image can be represented by a reflectance function $r(x, y)$, which assigns to any point $(x, y)$ of the image a value between 0 and 1 representing its light reflectance: 0 for black (i.e., no reflected light), 1 for white (i.e., full light reflectance), and intermediate values for in-between shades. In the case of transparencies, the reflectance function is replaced by a transmittance function defined in a similar way.

A superposition of such images can be obtained by overprinting, or by laying printed transparencies on top of each other. Since the superposition of black and any other shade always gives black, this suggests a multiplicative model for the superposition of monochrome images. Thus, when $m$ monochrome images are superposed, the reflectance of the resulting image is given by the product of the reflectance functions of the individual images:

$r(x, y)=r_{1}(x, y) r_{2}(x, y) \ldots r_{m}(x, y)$.

Let us now explain what we mean by periodic and aperiodic or stochastic layers. A function $f(x)$ is said to be periodic if there exists a nonzero number $p$, such that for any $x \in \mathbb{R}, f(x+p)=f(x)$. Similarly, a layer $r(x, y)$ is said to be periodic if there exists a nonzero vector $\mathbf{p}$ $=\left(p_{1}, p_{2}\right)$, such that for any $(x, y) \in \mathbb{R}^{2}, r\left(x+p_{1}, y+p_{2}\right)$ $=r(x, y)$. If there exist two independent vectors having this property, $r(x, y)$ is said to be two-fold periodic. A layer $r(x, y)$ is said to be aperiodic if it is not periodic. For example, the image of a human portrait or a natural landscape is aperiodic. As a second example, a random dot screen consisting of randomly positioned black dots is also aperiodic. Note, however, that this random dot screen may also be considered as a stochastic layer, from a more statistical point of view, if we consider the screen in question as just one possible realization of a stochastic process, having some given statistical distribution. In the case of random dot screens (or more generally, random scatter-see Chap. 17 in Ref. 6), the terms aperiodic layer, stochastic layer, and random layer are sometimes used interchangeably.

Finally, for the sake of simplicity we only consider layers having a uniform distribution of their microstructure elements (and hence a constant mean gray level), although our results hold also for more complex structures, such as halftone gradations, halftoned images with varying gray levels, etc.

\section{Superposition of Aperiodic Layers}

While the superposition of two identical periodic layers with a small angle or scaling difference generates moiré effects that are themselves periodic, the superposition of two identical aperiodic layers with a small angle or scaling difference generates an aperiodic moiré effect known as a Glass pattern [see Fig. 2(b)]. This moiré pattern is concentrated around a certain point in the superposition, and in contrary to periodic moirés, it gradually disappears as we go farther away from this point. Depending on whether it was obtained by rotation of one of the superposed layers, by a scaling transformation, or by a combination of both, it gives rise to an intriguing ordering of the microstructure elements in the superposition in "trajectories" having a circular, radial, or spiral shape [see Figs. 2(b), 3(a), and 3(b)]. Other layer transformations may give rise to Glass patterns having elliptic, hyperbolic, or other geometrically shaped trajectories. ${ }^{4}$ However, when we rotate one of the aperiodic layers by $180 \mathrm{deg}$ [see Fig. 2(c)], the Glass pattern completely disappears.

\subsection{Glass Patterns and Correlation}

As already explained by Glass, this phenomenon occurs thanks to the local correlation between the structures of the two superposed layers. In fact, its intensity can be used as a visual indication to the degree of correlation between the two layers in each point of the superposition. Thus, when two identical layers having the same arbitrary structure are slightly rotated on top of each other [see Fig. 2(b)], a visible Glass pattern is generated around the center of rotation, indicating the high correlation between the two layers in this area. Within the center of this Glass pattern the corresponding elements from both layers fall almost exactly on top of each other, but slightly away from the center they fall just next to each other, generating circular trajectories of point pairs. Further away from the center, the correlation between the two layers becomes smaller and smaller, and the elements from both layers fall in an arbitrary, noncorrelated manner. In this area the Glass pattern is no longer visible. This explains why the Glass pattern gradually decays and disappears as we go away from its center. Note, however, that when the two superposed layers are not at all correlated, no Glass pattern appears in the superposition [this is indeed what happens when we rotate one of the aperiodic transparencies by $180 \mathrm{deg}$, as shown in Fig. 2(c)]. In intermediate cases, where the two superposed layers are only partially correlated (for example, when one layer is a copy of the other with some percent of random noise being added), the Glass pattern is weaker and less perceptible, depending on the degree of the correlation which still remains between the superposed layers.

These facts are succinctly formulated by the following general rule.

Rule 1 (existence condition for Glass patterns): The superposition of layers gives rise to a Glass pattern iff $(=$ if and only if) there exists some degree of correlation between the superposed structures.

In fact, as we show in Sec. 3.3, the bright and dark areas that form the Glass pattern are due to variations in the correlation between the superposed layers. The particular case in which the correlation is constant throughout the superposition is explained in Sec. 3.2.

It should be noted that although this formulation of Rule 1 uses the term Glass patterns, it is in fact completely general, and it covers both periodic and aperiodic cases. As we see later, periodic cases are, indeed, particular cases of a general layer superposition, and in spite of their apparently different look, they still satisfy the same fundamental rules, just as any other layers. Simply, because of their additional internal structure, periodic cases also satisfy several additional specific rules (that are expressed in terms of periods or frequencies, as described by the classical periodic moiré 


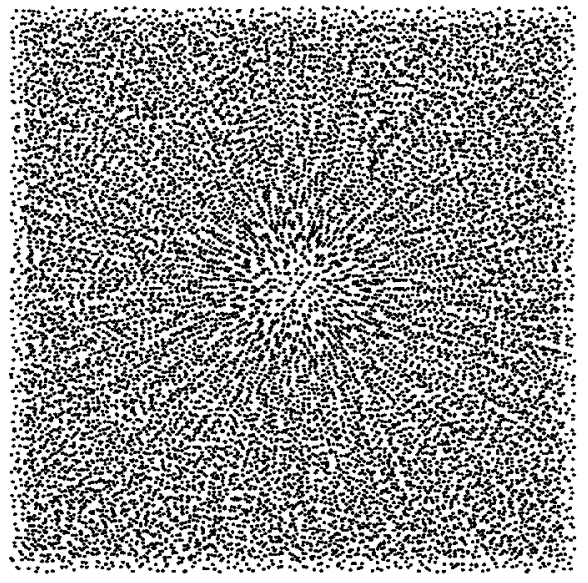

(a)

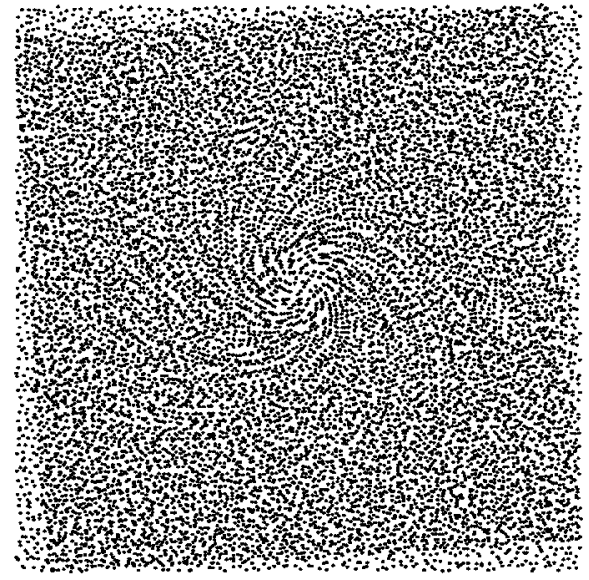

(b)

Fig. 3 (a) Same as in Fig. 2(b), but with a small scaling difference (rather than an angle difference) between the two layers. Note that in this case the microstructure consists of radial trajectories rather than concentric circular trajectories. (b) Same as in (a), but with both a small angle and a small scaling difference between the two identical layers. In this case the microstructure consists of spiral trajectories.

theory), rules that are no longer valid for general aperiodic cases.

\subsection{Stable Versus Singular Moiré-Free Superpositions}

Just as in the periodic case (see Sec. 2.9 in Ref. 2), we can distinguish in the general case, too, between two types of moire-free superpositions. Suppose that two identical layers, periodic or not, are superposed exactly one on top of the other, possibly with some fixed percent of random noise being added throughout. In this case the correlation between the layers remains constant throughout the superposition, and no macro-moiré effects are visible. Upon first observation, this situation resembles the moire-free case that occurs when the two superposed layers are completely independent of each other and have no correlation at all. However, a big difference exists between these two types of moiré-free superpositions. In the first case, the moire (or Glass) pattern does exist, but it is not visible because it is infinitely big; but such a moiré-free superposition is very unstable, since any slight deviation in the angle or in the scaling of any of the superposed layers may cause the moiré to come back from infinity and become clearly visible. This situation is called a singular moire-free superposition. On the other hand, moire-free superpositions, where the superposed layers are completely independent of each other, are stable moiré-free superpositions, and even when small angle or scale deviations occur between their individual layers, no macro-moiré effects become visible. This is, indeed, what is really meant by people saying that "the superposition of random screens does not generate moiré effects," as is often heard in the context of random screen halftoning, e.g., in color printing.

\subsection{Macrostructures and Microstructures in the Superposition}

As we can see, the explanation in Sec. 3.1 is based on an observation of the individual elements of the original layers and their behavior in the superposition. We say, therefore, that this explanation is based on the microstructure. To obtain the point of view of the macrostructure, we have to look at the layers and their superposition from a bigger distance, where the individual elements of the layers are no longer discerned by the eye, and what we see is only a gray-level average of the microstructure in each area of the superposition. From the point of view of the macrostructure, the center of the Glass pattern consists of a brighter gray level than areas farther away, due to the partial overlapping of the microstructure elements of both layers in this area. Farther away, elements from the two layers are more likely to fall side by side, thus increasing the covering rate and the macroscopic gray level. This means that the Glass pattern is not just an optical illusion, and it corresponds, indeed, to the physical reality. In fact, just as in the periodic case (see Proposition 8.1 in Ref. 2), moiré patterns are simply the macroscopic interpretation of the variations in the microstructures throughout the superposition.

Note that the ordering of the microstructure elements within a Glass pattern into circular, radial, or spiral trajectories is no longer visible from far away (try to observe Fig. 3 from a distance of 3 to $4 \mathrm{~m}$, where the individual elements of the layers are no longer discerned by the eye!). Therefore, these trajectories are not part of the macrostructure description, and they belong to the microstructure of the superposition, just as rosettes in the periodic case. And indeed, from the point of view of the macrostructure, there is no distinction between gray levels obtained when the neighboring elements in the superposition are located on circular trajectories, due to rotation, or on radial trajectories, due to a scaling transformation. What counts in both cases is the resulting mean coverage rate, which determines the overall gray level, and not the specific geometric arrangement.

It is interesting to note, as it is well known in the field of halftoning, that even in a superposition of uncorrelated random screens, where no macrostructures (Glass patterns) exist, the microstructure still may give rise to various random dot alignments such as nebulous or worm-like structures 


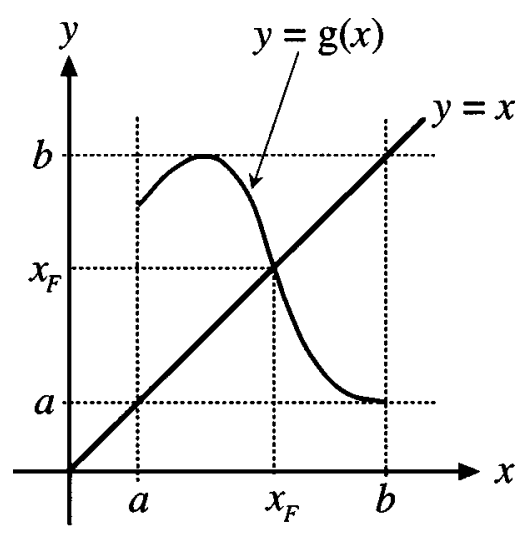

(a)

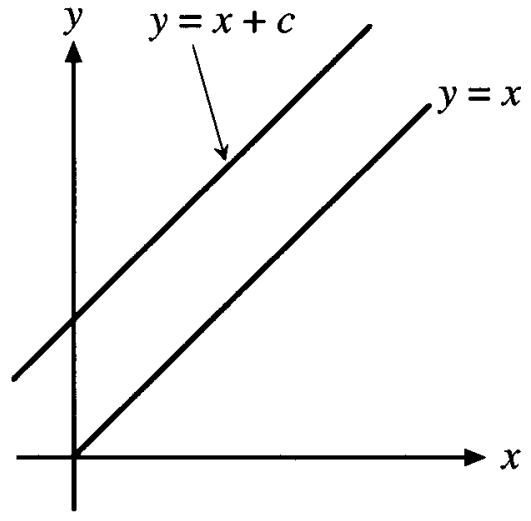

(b)

Fig. 4 (a) Illustration of the fixed point theorem in the 1-D case. Any continuous function $y=g(x)$ that maps a domain $D=[a, b]$ onto itself crosses the diagonal $y=x$ within the domain $[a, b]$ at least once. At each such point $x_{F}$ we have, therefore, $g\left(x_{F}\right)=x_{F}$. Moreover, due to the continuity of the function $g$, for any point $x_{G}$ within a near neighborhood of $x_{F}$, we have: $g\left(x_{G}\right) \approx x_{G}$. (b) The fixed point theorem is not generally valid when $D$ is the full range of $R$. This can be illustrated by any continuous function of the type $g(x)=x+c, c \neq 0$. Although these functions map $\mathrm{R}$ onto itself, they are parallel to the diagonal $y=x$, and hence they never cross it for any finite value $x_{F} \in \mathrm{R}$, meaning that for no point $x_{F} \in \mathbb{R}$ we have $g\left(x_{F}\right)=x_{F}$.

(artifacts). ${ }^{7}$ Even when these artifacts do not influence the macroscopic, overall gray level of the superposition (i.e., when they do not affect the average covering rate throughout the superposition), they still may be more or less conspicuous when viewed from a close distance, depending on the statistical nature or distribution of the points in the original screens (fully random, blue noise, ${ }^{8}$ green noise, ${ }^{9}$ etc.).

As we can see, just as in the case of periodic layers, the microstructures generated in the superposition of aperiodic layers may have very interesting and intriguing shapes, and their study is certainly not less fascinating than the study of the macrostructures. But although the investigation of the microstructures and their morphology in the superposition of aperiodic layers deserves full research on its own, in the present contribution we mainly concentrate on the investigation of the macrostructure aspects of the superposition and on its various mathematical properties. In the next sections we see what happens to the macrostructures in the superposition of periodic or aperiodic layers under layer transformations, and this investigation leads us to our second universal rule on layer superpositions.

\section{Fixed Point Theorem}

A famous theorem in mathematical topology, known as the fixed point theorem (Ref. 10, p. 653), says that any continuous function $g(x)$ that maps the domain $D=[a, b]$ onto itself, $g:[a, b] \rightarrow[a, b]$, has at least one fixed point in $[a, b]$ [namely, a point $x_{F} \in[a, b]$ that is mapped by $g(x)$ to itself: $\left.g\left(x_{F}\right)=x_{F}\right]$. This theorem is clearly illustrated in Fig. 4(a).

This fundamental theorem can be easily generalized to higher dimensions, although in such cases it can no longer be graphically illustrated as in Fig. 4(a). For example, a $2-\mathrm{D}$ version of the fixed point theorem states that any continuous mapping $\mathbf{g}(x, y)$ that maps the disk $D=\left\{(x, y) \mid x^{2}\right.$ $\left.+y^{2} \leqslant r\right\}$ into itself has at least one fixed point in $D$, namely, a point $\left(x_{F}, y_{F}\right) \in D$ that is mapped by $\mathbf{g}(x, y)$ to itself: $\mathbf{g}\left(x_{F}, y_{F}\right)=\left(x_{F}, y_{F}\right)$ (Ref. 10, p. 176).

It is interesting to note, however, that the fixed point theorem is not generally valid for infinite domains $D$ such as $D=\mathbb{R}$, or, in the $2-\mathrm{D}$ case, $D=\mathbb{R}^{2}$ (the entire $x, y$ plane). In such cases the theorem still holds for many functions $g$, but there exist other functions $g$ for which the theorem fails. This is illustrated, for the 1-D case, in Fig. 4(b). Although any function of the type $g(x)=x+c$ (with $c \neq 0)$ is continuous and fully maps $\mathbb{R}$ onto itself, there exist for these functions no fixed point $x_{F} \in \mathbb{R}$ such that $g\left(x_{F}\right)=x_{F}$ (unless we admit that parallel lines meet at infinity, in which case we may say that $x_{F}=\infty$ is a fixed point). * However, other continuous functions that map $\mathbb{R}$ onto itself, such as $g(x)=x^{3}$, do have fixed points, since they do cross the diagonal $y=x$ at least at one point $x_{F}$. A similar situation exists also in the 2-D case: While for many continuous mappings $\mathbf{g}(x, y)$ from $\mathrm{R}^{2}$ onto itself, such as scalings or rotations, there exists a fixed point, for other mappings such as translations $\mathbf{g}(x, y)=(x-a, y-b)$, there exist no fixed points (again, unless we consider infinity as a fixed point). However, the most important result for our needs may be formulated as follows.

The affine fixed point theorem: All nondegenerate affine mappings $\mathbf{g}(x, y)$ from $\mathbb{R}^{2}$ onto itself have a single fixed point.

This theorem asserts that all mappings such as rotations, scalings, etc., as well as their combinations, have indeed a fixed point. This also includes all of their combinations with translations, but pure translations are excluded. This theorem is explained and demonstrated in Appendix A.

\footnotetext{
*Note that the function $g(x)=x+c, c \neq 0$, is not a valid counter-example for the fixed point theorem with $D=[a, b]$, simply because it does not map $D$ onto itself.
} 
Let us see now how the fixed point theorem can help us formalize our subject of interest, the superposition of similar structures, periodic or not. This mathematical formalization will allow us in the following sections to deduce important facts on the behavior of Glass patterns. Suppose we are given a layer $r_{1}(x, y)$ consisting of an arbitrary structure, as explained in Sec. 2. We generate a second, slightly modified layer $r_{2}(x, y)$ by applying on $r_{1}(x, y)$ a continuous mapping (coordinate transformation) $\mathbf{g}(x, y)$ that maps the $x, y$ plane $\mathbb{R}^{2}$ onto itself. For example, $r_{2}(x, y)$ could be a slightly rotated version of $r_{1}(x, y)$. We now superpose the two layers $r_{1}(x, y)$ and $r_{2}(x, y)$, for example by overprinting, or by laying their transparencies on top of each other. The superposition thus obtained is represented mathematically by the product:

$r(x, y)=r_{1}(x, y) r_{2}(x, y)$.

Suppose that the continuous mapping $\mathbf{g}(x, y)$ has a fixed point $\left(x_{F}, y_{F}\right)$. This means that at the point $\left(x_{F}, y_{F}\right)$ we have $r_{2}\left(x_{F}, y_{F}\right)=r_{1}\left[\mathbf{g}\left(x_{F}, y_{F}\right)\right]=r_{1}\left(x_{F}, y_{F}\right)$, so that the point $\left(x_{F}, y_{F}, z_{F}\right)$ belonging to the surface $z=r_{1}(x, y)$ remains unchanged after applying the mapping $\mathbf{g}(x, y)$. For example, if it was a black point, it remains a black point in $r_{2}(x, y)$, and if it was a white point, it remains a white point in $r_{2}(x, y)$. Furthermore, in the neighborhood of this fixed point, any point $\left(x_{G}, y_{G}, z_{G}\right)$ of $r_{1}(x, y)$ has been only slightly displaced in $r_{2}(x, y)$. How does this affect the superposition of Eq. (2)?

Clearly, the superposition $r(x, y)$ is darker than each individual layer, since it becomes black wherever any of the superposed layers is black. However, the mean gray level of the superposition remains brighter in a close neighborhood around the fixed point $\left(x_{F}, y_{F}\right)$, since in this area the black dots of $r_{2}(x, y)$ fall almost exactly on top of their original counterparts in $r_{1}(x, y)$, so that the mean gray level is only slightly darker than in $r_{1}(x, y)$. But as we go farther from the fixed point $\left(x_{F}, y_{F}\right)$, the correlation between the dots of $r_{2}(x, y)$ and the dots of $r_{1}(x, y)$ gradually decreases, and consequently the mean gray level of the superposition becomes darker as the black points of $r_{2}(x, y)$ fall more often between black points of $r_{1}(x, y)$, leaving less white area in the superposition.

If the dots of $r_{1}(x, y)$ [and hence the dots of $r_{2}(x, y)$ ] are randomly distributed, then far away from the fixed point $\left(x_{F}, y_{F}\right)$ there will no longer be any correlation between the points of the two layers, and the resulting gray level in the superposition will remain constant as we go farther from $\left(x_{F}, y_{F}\right)$. However, if $r_{1}(x, y)$ is a periodic structure, such as a periodic dot screen, then as we go farther from the fixed point $\left(x_{F}, y_{F}\right)$, the mean gray level will periodically become darker and brighter, because zones of in-phase superposition, where elements of the two layers fall on top of each other, repeatedly alternate with zones of counterphase superposition, where elements of the two layers fall between each other [compare Figs. 2(b) and 2(d)]. It is interesting to note that in the superposition of partly random layers, such as periodic dot screens with a certain degree of randomness being added, the resulting Glass patterns have, indeed, an intermediate look. Depending on the case, they still may have around the center oscillations between darker and brighter areas, but if the correlation between the layers decreases with the distance, these oscillations gradually fade out and disappear as we go farther from the center of the Glass pattern.

This correspondence between Glass patterns and periodic moirés is further developed in the next section. We will see there that, in fact, periodic moirés are simply a particular case of Glass patterns that occurs when the superposed layers are periodic.

\section{Behavior of Glass Patterns and of Periodic Moirés Under Layer Mappings}

Having understood the mathematical meaning of Glass patterns, let us try to see their behavior when any of the superposed layers undergo a transformation such as rotation, scaling, translation, etc. Moreover, since the behavior of periodic moirés under such transformations is already fully known from the classical moire theory, it would be interesting to compare the behavior of both cases, periodic and aperiodic, and to see if they follow the same mathematical rules.

To study the behavior of a Glass pattern, we must, of course, make sure that a Glass pattern is indeed generated in our layer superposition. Therefore, we have to superpose layers that are sufficiently correlated. The easiest way of doing so is to assume a full correlation, i.e., that the superposed layers, periodic or not, be fully identical before the application of the layer mappings in question. In the rest of this section we make this assumption. Note that this does not cause a loss of generality, since in cases where the original layers are only partially correlated (for example, due to the presence of some random noise), the Glass patterns may look somewhat different or be less visible, but their behavior under layer mappings remains the same.

\subsection{Behavior Under Layer Rotations}

The simplest nontrivial layer transformation consists of a rotation of any of the superposed layers. This case has the practical advantage of being very easy to experiment by manipulating superposed transparencies. Suppose we have two identical transparencies consisting of the same arbitrary dot pattern, periodic or not. We superpose the two transparencies precisely on top of each other, and while keeping the first transparency (say, the upper one) fixed, we slightly rotate the other one by a small angle $\alpha$, so that a Glass pattern becomes visible around the fixed point at the rotation center. As we have already seen, the center of the Glass pattern is brighter than areas further away, due to the partial overlapping of the black elements of both layers around the fixed point. This behavior at the center is common to both periodic and stochastic cases, and indeed, the difference between these cases becomes apparent only farther away from the fixed point. In a stochastic case, as we go farther away from the fixed point, the mean gray level of the superposition is stabilized at a certain darker level [see Fig. 2(b)], because farther from the center the correlation between the two layers becomes negligible. But in a periodic case [see Fig. 2(d)], the brighter gray level at the center becomes alternately darker and brighter as we go away from the fixed point, and it continues to oscillate periodically, because zones of in-phase superposition, where ele- 
ments of the two layers fall on top of each other, repeatedly alternate with zones of counterphase superposition, where elements of the two layers fall between each other.

We may say, therefore, that the Glass pattern that is generated around the fixed point in a periodic case is periodic. However, from another point of view, we may say that while in the stochastic case there exists only one Glass pattern, which is located around the fixed point, in the periodic case, the Glass pattern that is generated around the fixed point is periodically repeated throughout the superposition, forming the bright areas of the periodic moire pattern. From this point of view, the periods of a periodic moire pattern are simply duplicates of the main Glass pattern that is generated around the fixed point, and the period length of the moire corresponds to the distance between these duplicates. ${ }^{\dagger}$ This does not mean, of course, that our rotation transformation $\mathbf{g}(x, y)$ has more fixed points when the two superposed layers are periodic than when the layers are aperiodic. Obviously, in both cases $\mathbf{g}(x, y)$ has exactly one fixed point. But when the two superposed layers are periodic, we also have infinitely many points of coincidence between the two superposed layers, where the two layers happen to coincide because of the periodicity in their internal structure. But these points of coincidence are not fixed points of the underlying mapping $\mathbf{g}(x, y)$. We can say, therefore, that the fixed point of $\mathbf{g}(x, y)$ determines the main periodic tile of the moire, while all the other periodic tiles are only duplicates that exist due to the periodicity of the superposed layers.

Note, however, that in spite of all these differences between the Glass patterns in periodic and aperiodic superpositions, their fundamental behavior under layer rotations remains basically the same. In both cases, when the angle $\alpha$ departs from 0 , the Glass pattern (respectively, the periodic tile of the moiré) becomes smaller and smaller until it completely disappears. And inversely, as the angle $\alpha$ tends to 0 , the Glass pattern (respectively, the periodic tile of the moiré) becomes bigger and bigger, until when $\alpha$ reaches 0 we obtain a singular superposition with an infinitely big moiré, which is no longer visible.

\subsection{Behavior Under Layer Scalings}

A similar effect occurs also in the case of a scaling transformation. Note, however, that in this case the visual study of the effect by using superposed transparencies is not as easy as in the case of rotation, because it is not possible to manually stretch or shrink transparencies. For testing this case one needs, therefore, to prepare in advance a set of reduced or enlarged copies of the original layer (for example, zoomed photocopies). A better solution would be to make simulations on a computer screen, since this would permit us to observe the superposition continuously while the scaling rate is gradually being varied.

\footnotetext{
It is important to note, however, that these duplicates are not necessarily identical in their microstructure, and the periodicity of the moire concerns only its macrostructure, namely, the moire intensity profile (the variation in the mean gray level that is observed from such a distance that the microstructure detail of the original layers is no longer discerned by the eye). In other words, although the microstructure in the superposition of two periodic layers is not always periodic, the intensity profile of the isolated moiré is, indeed, periodic (see Sec. 6.3 in Ref. 1).
}

Suppose we have two identical layers consisting of the same arbitrary dot pattern, periodic or not. We superpose the two layers precisely on top of each other, and while keeping the first layer fixed, we slightly scale the other one [see Fig. 3(a)]. Once again, a Glass pattern will become visible around the fixed point, whose center is brighter than areas farther away, due to the partial overlapping of the black elements of both layers around the fixed point. Although the microstructure obtained in this case is different than in the case of layer rotations [it consists of radial rather than circular dot trajectories, compare Figs. 3(a) and 2(b)], the macroscopic properties of the Glass pattern remain the same. And again, while in the stochastic case as we go farther from the fixed point, the mean gray level of the superposition is stabilized at a certain darker level, in the periodic case as we go farther from the fixed point, the brighter gray level at the center alternately becomes darker and brighter, and it continues oscillating repeatedly as the elements of the two layers periodically fall on top of each other (in phase) or between each other (in counterphase).

Thus, we may say, once again, that while in the stochastic case there exists only one Glass pattern, which is located around the fixed point, in the periodic case, the Glass pattern that is generated around the fixed point is periodically repeated throughout the superposition, forming the bright areas of the periodic moire pattern.

But just as we have seen with layer rotations, in spite of the difference between the Glass patterns in periodic and aperiodic superpositions, their fundamental behavior under layer scalings remains basically the same. In both cases, when the scaling factor $s$ gradually departs from 1, the Glass pattern (respectively, the periodic tile of the moiré) becomes smaller and smaller. And inversely, as the scaling factor $s$ tends to 1, the Glass pattern (respectively, the periodic tile of the moiré) becomes bigger and bigger, until when $s$ reaches 1 we obtain a singular superposition with an infinitely big moiré, which is no longer visible. It should be mentioned, however, that while in the periodic case new higher order moirés may occur around $s=2,3$, or $s$ $=1 / 2,1 / 3$, etc., in the purely stochastic case, no higher order moirés exist, since at such scaling values no correlation exists between the superposed layers [for instance, a random screen $r(x, y)$ is not correlated with $r(2 x, 2 y)]$.

\subsection{Behavior Under Layer Shifts}

Suppose we have two identical transparencies that are superposed with a small angle difference (or a small scaling difference), so that a visible Glass pattern is generated around the fixed point. What happens to this Glass pattern when we laterally translate one of the transparencies with respect to the other? The answer for the case of periodic moirés is already well known: the moiré pattern will simply be translated (by a much longer distance than the original layer shift), without undergoing any other modifications. The extent and the direction of its translation are determined by the extent and the direction of the shifts in the original layers as explained, for example, in Sec. 7.6 of Ref. 2 (see Fig. 5). Since the superposition of periodic layers is a particular case of the superposition of any general layers, it would be reasonable to expect that the behavior of a periodic moiré under layer shifts should be a particular case of the behavior of a Glass pattern under the same layer 


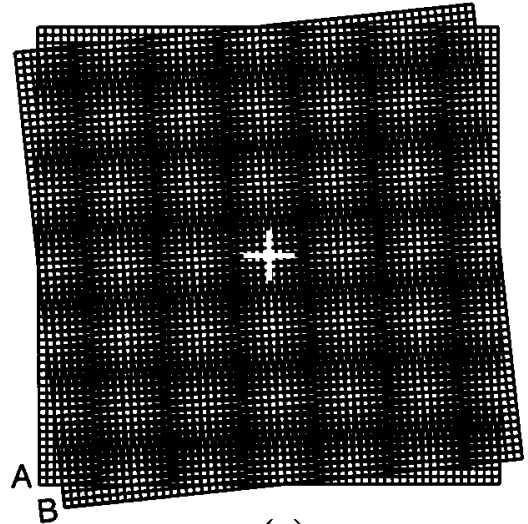

(a)

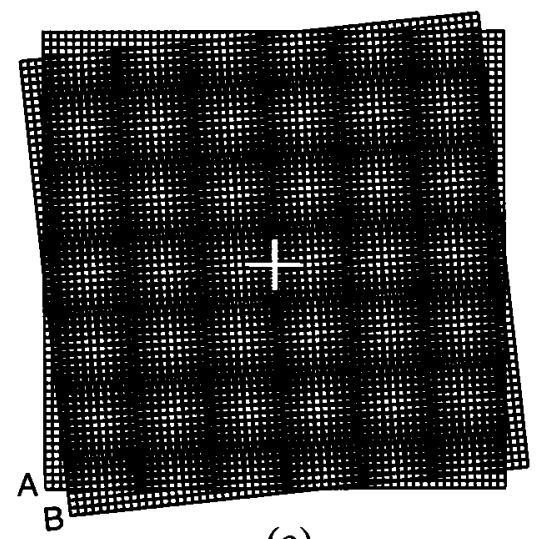

(c)

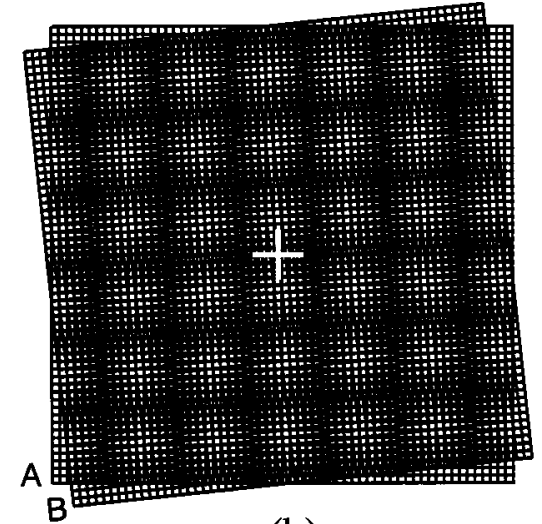

(b)

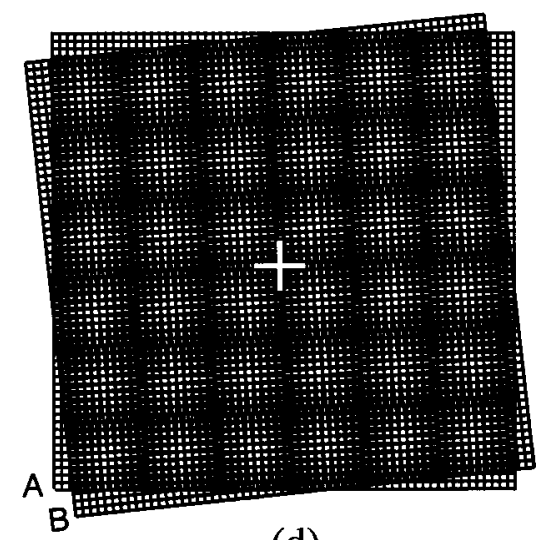

(d)

Fig. 5 A periodic moiré between two identical periodic grids of period $T$ that are superposed with a small angle difference $\alpha$, and its behavior under layer shifts. The origin of each image is indicated by a cross. (a) Both grids and the resulting moiré are centered on the origin, in their initial position. (b) Grid $A$ is shifted by $1 / 2$ period (i.e., by $T / 2$ ) to the right. Consequently, the moire is shifted $1 / 2$ moiré period downward. (c) Grid $A$ is shifted by $1 / 2$ period (i.e., by $T / 2$ ) upward. Consequently, the moire is shifted $1 / 2$ moire period to the right. (d) Grid $A$ is shifted by $1 / 2$ period to the right and $1 / 2$ period upward. Consequently, the moire is shifted $1 / 2$ moire period downward and $1 / 2$ moiré period to the right.

shifts. And indeed, as described in detail next, simple experimentation with two superposed transparencies shows that exactly the same results are obtained in the periodic and aperiodic cases.

Suppose that a Glass pattern (respectively, a periodic moire pattern) is generated around the fixed point by rotating the second transparency by a small angle $\alpha$ counterclockwise, and that we slightly shift the first transparency (the nonrotated layer) in a given direction. ${ }^{*}$ As shown in Figs. 5 and 6, the resulting effect will be a much larger shift of the Glass pattern (respectively, the periodic moiré), in a direction that is basically perpendicular to the shift of the first transparency.

- When the first transparency is slightly shifted to the right, the Glass (or moiré) pattern largely moves downward.

\footnotetext{
We choose this layer convention to remain compatible with the figures and the examples given for the periodic case in Chap. 7 of Ref.1, some of which are reproduced here.
}

- When the first transparency is slightly shifted to the left, the Glass (or moiré) pattern largely moves upward.

- When the first transparency is slightly shifted upward, the Glass (or moiré) pattern largely moves to the right.

- And when the first transparency is slightly shifted downward, the Glass (or moiré) pattern largely moves to the left.

The identical qualitative behavior of both periodic and aperiodic cases further confirms our assumption that both cases are, indeed, two different facets of the same phenomenon. But if our assumption is correct, the behavior of both cases must be identical quantitatively, too. Since the quantitative behavior of the periodic moire under layer shifts is already well known (see Sec. 7.6 in Ref. 2), we try now to determine quantitatively the behavior of the aperiodic case (i.e., the shift of the Glass pattern), to see if we obtain the same results.

In order to do so, let us try to locate the fixed point (i.e., the center of the Glass pattern) when the second layer is 


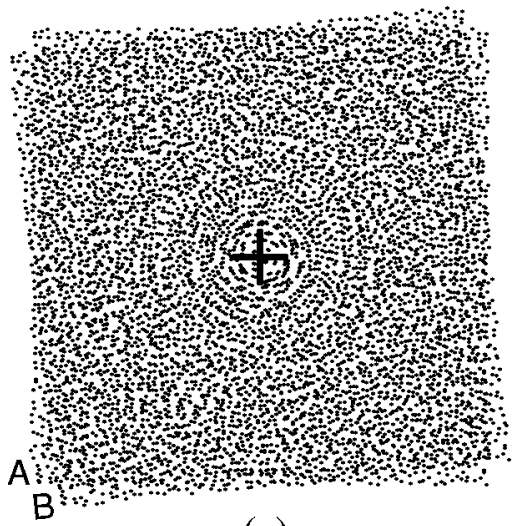

(a)

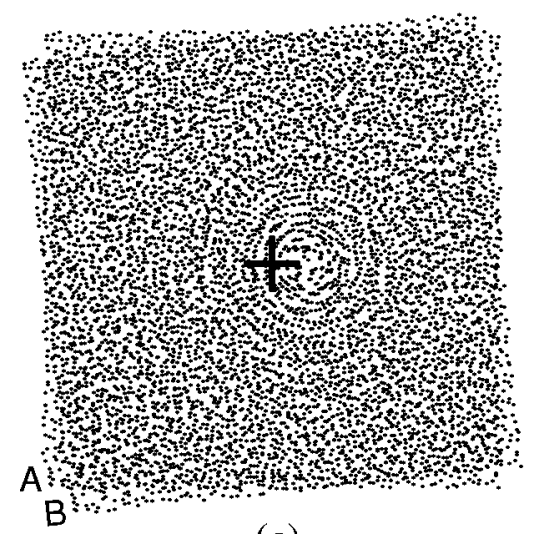

(c)

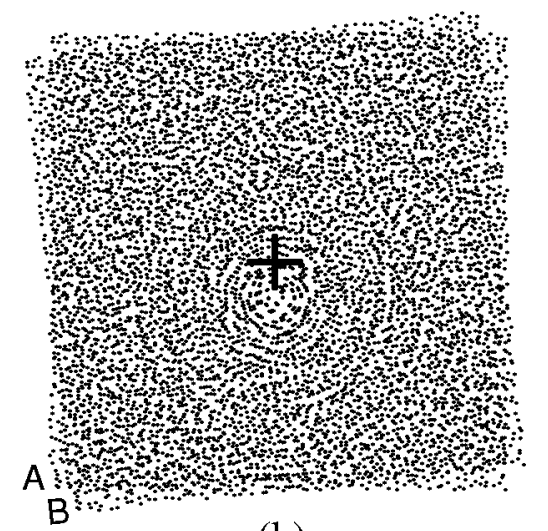

(b)

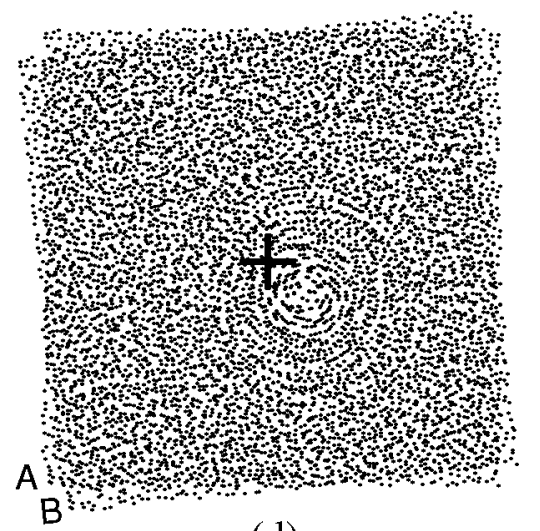

(d)

Fig. 6 A Glass pattern between two identical aperiodic dot screens that are superposed with a small angle difference $\alpha$, and its behavior under layer shifts. The origin of each image is indicated by a cross. (a) Both layers and the resulting Glass pattern are centered on the origin, in their initial position. (b) Layer $A$ is shifted by $x_{0}$ to the right. Consequently, the Glass pattern is shifted downward. (c) Layer A is shifted by $y_{0}$ upward. Consequently, the Glass pattern is shifted to the right. (d) Layer $A$ is shifted by $x_{0}$ to the right and by $y_{0}$ upward. Consequently, the Glass pattern is shifted downward and to the right. Note that the angle difference $\alpha$ between the superposed layers is the same as in Fig. 5, and the layer shifts are also the same as in Fig. 5: $x_{0}=T / 2, y_{0}=T / 2$. And indeed, the resulting shift of the Glass pattern is the same as the resulting shift of the moire pattern in Fig. 5 (although in the aperiodic case it cannot be expressed in terms of moiré periods).

rotated by angle $\alpha$, and the first, unrotated layer is shifted laterally in the original $x$ and $y$ directions by $\left(x_{0}, y_{0}\right)$. This is also equivalent to rotating the second layer by angle $\alpha$, and then shifting it along the original $x$ and $y$ directions by $-\left(x_{0}, y_{0}\right)$, while the first layer remains fixed. The mapping $\mathbf{g}(x, y)$ is given, therefore, by:

$x^{\prime}=x \cos \alpha+y \sin \alpha+x_{0}$,

$y^{\prime}=-x \sin \alpha+y \cos \alpha+y_{0}$

where $x, y$ are the coordinates before applying the rotation and the shift, and $x^{\prime}, y^{\prime}$ are the coordinates after the mapping. Our problem of finding the fixed point of $\mathbf{g}(x, y)$ consists, therefore, of finding when $\left(x^{\prime}, y^{\prime}\right)$ equals $(x, y)$. This happens, of course, where:

$x=x \cos \alpha+y \sin \alpha+x_{0}$

$y=-x \sin \alpha+y \cos \alpha+y_{0}$, which gives us the following linear set of equations for $x$ and $y$ :

$x(1-\cos \alpha)-y \sin \alpha=x_{0}$,

$x \sin \alpha+y(1-\cos \alpha)=y_{0}$.

It can be easily shown (for example, using Cramer's rule) that the solution of this set of equations is given by:

$x=\frac{x_{0}(1-\cos \alpha)+y_{0} \sin \alpha}{2(1-\cos \alpha)}$

$y=\frac{-x_{0} \sin \alpha+y_{0}(1-\cos \alpha)}{2(1-\cos \alpha)}$, 


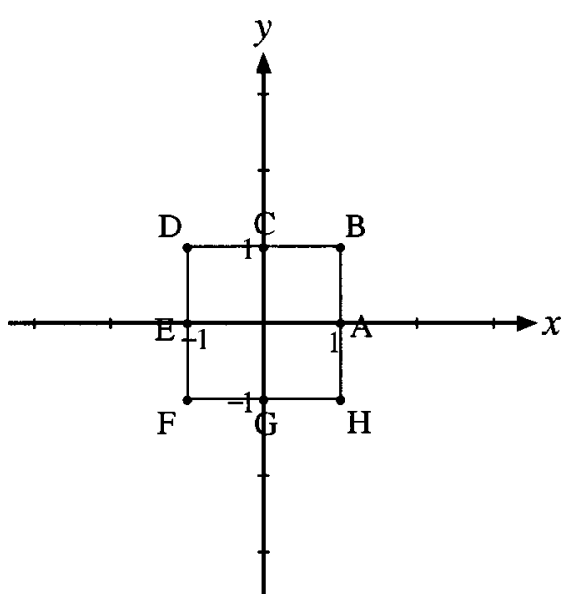

(a)

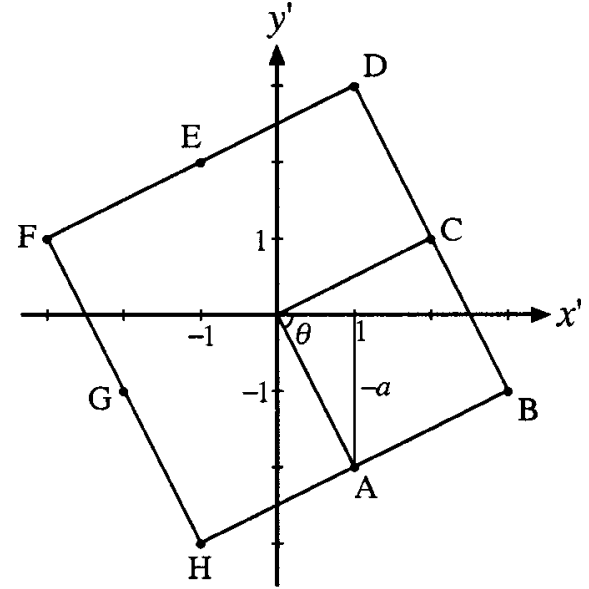

(b)

Fig. 7 Geometrical interpretation of the 2-D linear transformation, whose matrix is $\left(\begin{array}{cc}1 & a \\ -a & 1\end{array}\right)$. (a) shows points $A=(1,0), B=(1,1), C=(0,1), \ldots, H=(1,-1)$ in the $x, y$ plane before applying this transformation, and (b) shows the new locations of these points in the $x^{\prime}, y^{\prime}$ plane after the application of the transformation. For example, point $A$, whose initial coordinates in $(a)$ are $(1,0)$ is mapped by the transformation to the point $\left(\begin{array}{cc}1 & a \\ -a & 1\end{array}\right)\left(\begin{array}{l}1 \\ 0\end{array}\right)=\left(\begin{array}{c}1 \\ -a\end{array}\right)$ in (b).

or in matrix form:

$$
\begin{aligned}
\left(\begin{array}{l}
x \\
y
\end{array}\right) & =\frac{1}{2}\left(\begin{array}{cc}
1 & \frac{\sin \alpha}{1-\cos \alpha} \\
-\frac{\sin \alpha}{1-\cos \alpha} & 1
\end{array}\right)\left(\begin{array}{l}
x_{0} \\
y_{0}
\end{array}\right) \\
& =\frac{1}{2}\left(\begin{array}{cc}
1 & \cot (\alpha / 2) \\
-\cot (\alpha / 2) & 1
\end{array}\right)\left(\begin{array}{l}
x_{0} \\
y_{0}
\end{array}\right) .
\end{aligned}
$$

Note that this solution (fixed point) exists and is unique whenever $2(1-\cos \alpha) \neq 0$, i.e., whenever $\alpha \neq 0$.

Equation (7) means that the fixed point $(x, y)$ is simply a linear transformation of the lateral shift $\left(x_{0}, y_{0}\right)$ undergone by the original layer. The matrix of this transformation has the form of $\left(\begin{array}{cc}1 & a \\ -a & 1\end{array}\right)$ with $a=\cot (\alpha / 2)$. As shown in Fig. 7, this transformation corresponds, in fact, to:

- a rotation by angle $\theta$, where $\tan \theta=-a$, i.e., $\theta$ $=-\arctan a$

- and a scaling by factor $s: s=\left(1+a^{2}\right)^{1 / 2}$.

By inserting here $a=\cot (\alpha / 2)$ and remembering the factor $1 / 2$ before the matrix, we see that the location $(x, y)$ of the fixed point is obtained from the layer shift $\left(x_{0}, y_{0}\right)$ by a rotation of:

$\theta=-\arctan \left(\cot \frac{\alpha}{2}\right)=\operatorname{arccot}\left(\cot \frac{\alpha}{2}\right)-\frac{\pi}{2}=\frac{\alpha}{2}-\frac{\pi}{2}$,

and a scaling by:

$s=\frac{1}{2}\left[1+\cot ^{2}(\alpha / 2)\right]^{1 / 2}=\frac{1}{2 \sin (\alpha / 2)}$.
And indeed, as we have expected, this quantitative result fully corresponds to the moire shift obtained in the case of a periodic moiré:

As predicted by proposition 7.2 in Ref. 2, the moire in the periodic case is shifted in its own direction, which is, as shown in Fig. 4.8 of Ref. 2, reproduced here as Fig. 8, exactly $\alpha / 2-\pi / 2$.

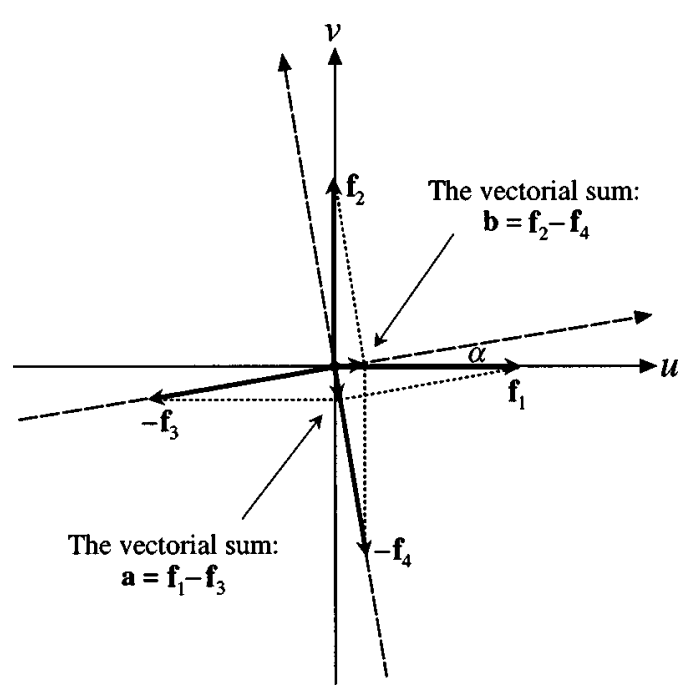

Fig. 8 Vector diagram explaining the moire orientation in the superposition of two identical periodic screens with an angle difference of $\alpha$. Vectors $\mathbf{f}_{1}$ and $\mathbf{f}_{2}$ are the main and secondary frequency vectors of the first layer, and $\mathbf{f}_{3}$ and $\mathbf{f}_{4}$ are the main and secondary frequency vectors of the second layer (note that in the figure we rather show $-\mathbf{f}_{3}$ and $-\mathbf{f}_{4}$, in the opposite directions). The moire effect generated in the superposition is represented by the vectors $\mathbf{a}=\mathbf{f}_{1}$ $-f_{3}$ and $b=f_{2}-f_{4}$. The orientation of the moiré, represented by the orientation of its main frequency vector $\mathbf{a}$, is $\alpha / 2-\pi / 2$. Namely, it is perpendicular to the bisector between the directions of the two original layers. 
Furthermore, the extent of the shift of the periodic moire is given, in terms of periods, by Eqs. (7.26) and (2.10) in Ref. 2, namely:

$b_{M}=T_{M}\left(\phi_{1}-\phi_{2}\right)=\frac{T}{2 \sin (\alpha / 2)}\left(\phi_{1}-\phi_{2}\right)$,

where $b_{M}$ is the resulting shift of the moire, $T_{M}$ is the period of the moire, $T$ is the period of the original layers, and $\phi_{1}$ and $\phi_{2}$ are the shifts of the original layers in terms of periods $T$. Noting that in our case $\phi_{2}=0$ (the second layer is not shifted), we see that for a shift of $d$ in the first layer (i.e., $\phi_{1}=d / T$ periods), the extent of the resulting shift of the moire is:

$b_{M}=\frac{1}{2 \sin (\alpha / 2)} d$.

Hence, the shift of the periodic moire is obtained by scaling up the shift $d$ of the original layer by the factor $s$ $=1 / 2 \sin (\alpha / 2)$, exactly as predicted by Eq. (9) according to fixed point considerations.

We see, therefore, that the resulting moire shifts in the periodic case and in the aperiodic case are indeed identical, and both are explained as a shift of the fixed point. However, in the periodic case, the same result can also be obtained in terms of periods, frequencies, Fourier series developments, etc., as was done in Ref. 2. This period- or frequency-based interpretation is extremely useful due to the new insights and powerful tools it offers for analyzing the periodic case. But in the general aperiodic case, we can no longer use period-based approaches, and we revert to the more general analysis in terms of the fixed point theorem.

\subsection{Behavior Under a General Affine Transformation}

In the most general affine case, when the transformation $\mathbf{g}(x, y)$ is given by:

$x^{\prime}=a_{1} x+b_{1} y+x_{0}$,

$y^{\prime}=a_{2} x+b_{2} y+y_{0}$,

the fixed point is given by the set of equations:

$$
\begin{aligned}
& \left(1-a_{1}\right) x-b_{1} y=x_{0}, \\
& -a_{2} x+\left(1-b_{2}\right) y=y_{0},
\end{aligned}
$$

whose solution is:

$$
\begin{aligned}
& x=\frac{\left(1-b_{2}\right) x_{0}+b_{1} y_{0}}{1-a_{1}-b_{2}+a_{1} b_{2}-a_{2} b_{1}}, \\
& y=\frac{a_{2} x_{0}+\left(1-a_{1}\right) y_{0}}{1-a_{1}-b_{2}+a_{1} b_{2}-a_{2} b_{1}},
\end{aligned}
$$

or in matrix form:

$$
\left(\begin{array}{l}
x \\
y
\end{array}\right)=\frac{1}{1-a_{1}-b_{2}+a_{1} b_{2}-a_{2} b_{1}}\left(\begin{array}{cc}
1-b_{2} & b_{1} \\
a_{2} & 1-a_{1}
\end{array}\right)\left(\begin{array}{l}
x_{0} \\
y_{0}
\end{array}\right) .
$$

This means that even in the general case where the layer transformation is given by the affine mapping $\mathbf{g}(x, y)$ of Eq. (12), the location $(x, y)$ of the fixed point is still a linear transformation of the shift $\left(x_{0}, y_{0}\right)$ undergone by the original layer.

It should be mentioned that this solution (i.e., the fixed point) exists and is unique iff the determinant of the homogenous equations in Eqs. (13), i.e., the denominator of Eq. (15), is nonzero:

$1-a_{1}-b_{2}+a_{1} b_{2}-a_{2} b_{1} \neq 0$.

If this condition is not satisfied, then either there exists no solution (fixed point) at all [this happens, for example, when $\mathbf{g}(x, y)$ is a pure translation], or there exists an infinity of solutions [for example: if $\mathbf{g}(x, y)$ consists of flipping over the $x$ axis, possibly followed by a vertical shift of $\left(0, y_{0}\right)$, then there exists an infinity of fixed points forming together a full horizontal line]. This subject is fully explained in Appendix A.

\subsection{Behavior Under General Layer Transformations}

Similar considerations also hold for more general layer transformations, such as second-order polynomial transformations, logarithmic transformations, etc. However, finding the fixed points in such cases may require some more complex calculations, using either analytic or numeric methods. Note that in the most general case there are more possible configurations for the fixed points than in an affine transformation. A general mapping may have, for example, no fixed points at all, one isolated fixed point, several isolated fixed points, or even a curved line consisting of fixed points.

In conclusion, we see that the following universal rule holds for periodic as well as aperiodic layers, and explains their fundamental behavior.

Rule 2 (moiré behavior rule): The behavior of the moiré under rotations, scalings and shifts of the individual layers (or more generally, under any layer mappings, linear or not), is determined by the fixed points of the mappings and their properties.

As we can see, our two universal rules determine the conditions for the generation of moiré effects, as well as the behavior of these moiré effects under rotations, scalings, shifts, or any other layer transformations. This gives us, indeed, a unified approach, which explains the fundamental properties of all types of moiré effects, periodic or aperiodic.

\section{Conclusions}

Because moiré effects are so frequently encountered in electronic imaging, a full understanding of their different forms is essential. In the present contribution we have gone a further step in this direction. We show that in spite of their different appearance, moiré effects that occur between 
periodic or aperiodic layers are, in fact, particular cases of the same basic phenomenon, and all of them satisfy the same fundamental, universal rules.

Rule 1 (moiré existence rule): The superposition of layers gives rise to a moiré pattern iff there exists some degree of correlation between the superposed structures.

Rule 2 (moiré behavior rule): The behavior of the moiré under rotations, scalings, and shifts of the individual layers (or more generally, under any layer mappings, linear or not), is determined by the fixed points of the mappings and their properties.

The first rule gives the conditions for the appearance of moire effects, and the second rule determines the behavior of these moiré effects under any layer transformations. Based on these general rules, we have presented a unified approach that explains the basic properties common to all the different types of moiré effects: moirés between stochastic, periodic, or repetitive layers. It is clear, however, that when additional structural information on the original layers exists, like in periodic or in repetitive cases, this additional information may allow the investigation of the moiré effects by means of more sophisticated methods, such as the Fourier theory, indicial equations, etc., thus offering further insights and analysis tools that are proper to such cases. But in the most general aperiodic or stochastic cases, we can no longer use period-based approaches, and we revert to the most basic interpretation of the moire phenomena as Glass patterns, and to their explanation by our universal rules.

The unified approach we have presented here is completely general, and it covers all the different types of moiré effects between any superposed layers. But it also offers us a new, interesting point of view on the classical moiré effects between periodic layers. While in a stochastic case (if we assume a nondegenerate affine layer transformation), there may exist at most one Glass pattern, which is located around the fixed point, in a periodic case, the Glass pattern that is generated around the fixed point is periodically repeated throughout the superposition, with its center forming the bright areas of the periodic moire pattern. From this point of view, the 2-D periods of a periodic moire pattern are simply duplicates of the main Glass pattern, which is generated around the fixed point, and the period length of the moiré corresponds to the distance between these duplicates.

Finally, although we mainly mention here superpositions of two layers, the results presented in this work are completely general and can be easily extended to superpositions of three or more layers or to the multichromatic case.

\section{Appendix A: Affine Fixed Point Theorem}

As we have seen in Sec. 4, the affine fixed point theorem states that all nondegenerate affine mappings $\mathbf{g}(x, y)$ from $\mathbb{R}^{2}$ onto itself have a single fixed point.

To better understand this theorem, let us analyze the different possible types of affine mappings $\mathbf{g}(x, y)$. The most general affine mapping is given by:

$x^{\prime}=a_{1} x+b_{1} y+x_{0}$,

$y^{\prime}=a_{2} x+b_{2} y+y_{0}$.
First, we consider the homogeneous mapping that is associated with $\mathbf{g}(x, y)$, i.e., the corresponding linear transformation where the shift $\left(x_{0}, y_{0}\right)$ is zero:

$x^{\prime}=a_{1} x+b_{1} y$

$y^{\prime}=a_{2} x+b_{2} y$.

Such a linear transformation may either:

a. map $R^{2}$ onto the whole of $R^{2}$ (this occurs, for example, in rotations, scalings, flipping over an axis, etc.)

b. map $\mathbb{R}^{2}$ onto $\mathbb{R}$ (for example, projection on the $x$ axis, etc.)

c. map $\mathbb{R}^{2}$ onto the origin $(0,0)$ [this occurs in the zero transformation that maps all the points $(x, y)$ to $(0,0)]$.

Cases $\mathbf{b}$ and $\mathbf{c}$ occur when the linear transformation in Eq. (18) is singular, i.e., when its determinant equals zero:

$\left|\begin{array}{ll}a_{1} & b_{1} \\ a_{2} & b_{2}\end{array}\right|=a_{1} b_{2}-a_{2} b_{1}=0$.

Such cases do not interest us, of course, and we are only interested in transformations belonging to type a, namely, when Eq. (18) is nonsingular.

But this is not yet all. Each such nonsingular linear transformation may either:

1. have a single fixed point, located at the origin (this occurs, for example, in rotations, scalings, etc.)

2. have a full line of fixed points that passes through the origin (for example, flipping over the $x$ axis, or scaling in the $y$ direction alone, have all points of the $x$ axis as fixed points)

3. have the full $x, y$ plane as fixed points (this occurs in the identity transformation).

The fixed points of transformation (18) are those points of the plane for which $\left(x^{\prime}, y^{\prime}\right)$ equal $(x, y)$, namely:

$x=a_{1} x+b_{1} y$,

$y=a_{2} x+b_{2} y$.

This gives us the following linear set of equations for $x$ and $y$ :

$\left(1-a_{1}\right) x-b_{1} y=0$,
$-a_{2} x+\left(1-b_{2}\right) y=0$.

Clearly, cases 2. and 3. occur when this linear set of equations is singular, i.e., when:

$\left|\begin{array}{cc}1-a_{1} & -b_{1} \\ -a_{2} & 1-b_{2}\end{array}\right|=1-a_{1}-b_{2}+a_{1} b_{2}-a_{2} b_{1}=0$ 
Once again, such cases do not interest us, and we are only interested in transformations belonging to type 1, namely, when Eq. (20) is nonsingular.

Consequently, we only consider transformations that satisfy conditions a and 1, i.e., where both of the determinants in Eqs. (19) and (21) are nonzero. We call such linear transformations nondegenerate linear transformations, and their associated affine mappings that are obtained by adding a shift of $\left(x_{0}, y_{0}\right)$ are called nondegenerate affine mappings.

It is clear, therefore, that all nondegenerate affine mappings $\mathbf{g}(x, y)$ from $\mathrm{R}^{2}$ onto itself have a single fixed point. This is, indeed, precisely what is claimed by our affine fixed point theorem.

As an illustration, let us mention that mappings such as rotations, scalings, etc. as well as their combinations have, indeed, a fixed point. This is also true for all of their combinations with translation, but not for pure translations. Note that pure translations are excluded, since their determinant in Eq. (21) is zero. In fact, the homogeneous transformation (18) that is associated with a pure translation is the identity transformation, that belongs to class 3 and has the whole $x, y$ plane as fixed points. But the addition of a translation destroys all of these fixed points, so that a pure translation has no fixed points.

As a final example, let us consider the linear transformation which consists of vertical scaling. This transformation belongs to class 2, and has the full $x$ axis as fixed points. What happens now when we add to this linear transformation a translation? In this case, the answer depends on the direction of the translation: If the translation is horizontal, it is clear that all the fixed points on the $x$ axis are destroyed, and the resulting affine mapping $\mathbf{g}(x, y)$ has no fixed points. But if the translation is vertical, the resulting affine mapping $\mathbf{g}(x, y)$ will still have a full line of fixed points, which is parallel to the $x$ axis. Note, however, that such cases are not treated by our affine fixed point theorem, since their determinant in Eq. (21) is zero. This theorem only considers nondegenerate affine mappings, but it does not say anything about degenerate affine mappings. In fact, as we have just seen, some degenerate affine mappings have a full line of fixed points, while others (such as pure translations) have no fixed points at all.

Note that it is possible to formulate a more general version of this theorem that treats all affine mappings from $\mathbb{R}^{2}$ onto itself, including degenerate cases such as vertical scalings and translations:

The generalized affine fixed point theorem: An affine mapping $\mathbf{g}(x, y)$ from $\mathbb{R}^{2}$ onto itself has a fixed point (either one or infinitely many) iff $\operatorname{rank} \mathbf{A}=\operatorname{rank} \mathbf{B}$, where $\mathbf{A}$ is the
$2 \times 2$ coefficient matrix of the homogeneous system of Eq. (20) and $\mathbf{B}$ is the $2 \times 3$ extended matrix that includes $x_{0}$ and $y_{0}$ in its third column:

$\mathbf{A}=\left(\begin{array}{cc}1-a_{1} & -b_{1} \\ -a_{2} & 1-b_{2}\end{array}\right) \quad \mathbf{B}=\left(\begin{array}{ccc}1-a_{1} & -b_{1} & x_{0} \\ -a_{2} & 1-b_{2} & y_{0}\end{array}\right)$

Moreover, if the rank of both $\mathbf{A}$ and $\mathbf{B}$ is 2, the fixed point is unique; if their rank is 1 , there exists a full line of fixed points; and if their rank is 0 , all the points of the $x, y$ plane are fixed points of $\mathbf{g}(x, y)$ [this occurs if $\mathbf{g}(x, y)$ is the identity mapping without translation].

This generalized theorem is, in fact, an application to the particular case of Eq. (20) of the algebraic theorem on the dimension of the solution space of a system of linear equations (Ref. 11, p. 143).

\section{References}

1. J. A. C. Yule, Principles of Color Reproduction, Wiley and Sons, New York (1967).

2. I. Amidror, The Theory of the Moiré Phenomenon, Kluwer, Dordrecht (2000).

3. L. Glass, "Moiré effect from random dots," Nature (London) 223 , $578-580$ (1969).

4. L. Glass and R. Pérez, "Perception of random dot interference patterns," Nature (London) 246, 360-362 (1973).

5. L. Glass, "Looking at dots," Math. Intell. 24, 37-43 (2002).

6. R. N. Bracewell, Two Dimensional Imaging, Prentice Hall, Englewood Cliffs, NJ (1995)

7. D. L. Lau, A. M. Khan, and G. R. Arce, "Stochastic moiré," Proc. IS\&T's PICS Conf. pp. 96-100 (2001)

8. R. Ulichney, "Dithering with blue noise," Proc. IEEE 76, 56-79 (1988).

9. D. L. Lau, G. R. Arce, and N. C. Gallagher, "Green-noise digital halftoning," Proc. IEEE 86, 2424-2444 (1998).

10. E. W. Weisstein, CRC Concise Encyclopedia of Mathematics, CRC, Boca Raton, FL (1999).

11. I. N. Bronshtein and K. A. Semendyayev, Handbook of Mathematics. Springer, Berlin, 1997.

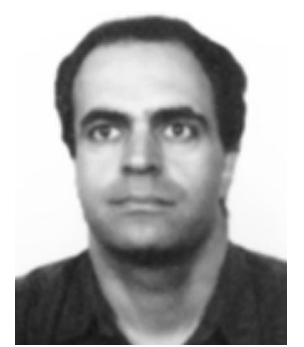

Isaac Amidror received his BSc degree in mathematics from the Hebrew University of Jerusalem, Israel, and his MSc degree in computer science from the Weizmann Institute of Science in Rehovot, Israel. He received a Japanese government scholarship for a two-year research period in the computer science department of the Toyohashi University of Technology in Japan. After having worked a few years in industry (notably in the fields of laser printing and digital typography), he received his PhD degree in the Swiss Federal Institute of Technology, in Lausanne, Switzerland. He has published numerous scientific papers and is the author of a recent book on the theory of the moire phenomenon (http://lspwww.epfl.ch/ books/moire/). His research interests include the mathematical foundations of moire phenomena, document security, color image reproduction, image processing, and digital typography. 